\title{
Insights into the Molecular Mechanism underlying Polymorph Selection.
}

\author{
Caroline Desgranges and Jerome Delhommelle
}

\section{Supporting Information}

\section{Expression and units used for the Yukawa (screened-Coulomb) potential}

The phase behavior of Yukawa systems is generally expressed in terms of dimensionless screening and temperature parameters.

If we take $a=n^{-1 / 3}$, where $n=N / V$ is the macroion number density, as the unit of length, then the Yukawa potential can be written as

$$
U(r)=\frac{\Phi}{a} \frac{e^{-\lambda r / a}}{r / a}
$$

where $\lambda$ is the dimensionless screening parameter.

The limits $\lambda \rightarrow 0$ and $\lambda \rightarrow \infty$ correspond to the one-component plasma and hard-sphere systems respectively. Following Robbins et al. (Robbins, M. O.; Kremer, K.; Grest, G. S. J. Chem. Phys. 1988, 88, 3286), we take the period of an Eistein oscillator in a crystal, $\tau_{E}$, as the unit of time, the Einstein phonon $m \omega_{E}^{2} a^{2}\left(\omega_{E}=2 \pi / \tau_{E}\right)$, as the unit of energy and define the dimensionless temperature $\tilde{T}=\frac{k_{B} T}{m \omega_{E}^{2} a^{2}}$, using the fcc values for the phonon energy by Robbins et al.

We consider two different values, $\lambda=5$ and $\lambda=10$, for the dimensionless screening parameter. For these two values of $\lambda$, we study the crystal nucleation and growth from the supercooled liquid. We choose the same degree of supercooling for both systems. The two systems are cooled at a temperature $25 \%$ below their respective melting temperature $\left(\tilde{T}=2.594 \times 10^{-3}\right.$ for $\lambda=5$ and $\tilde{T}=3.319 \times 10^{-3}$ for $\lambda=10$ according to the equation for the melting line proposed by Robbins et al. (Robbins, M. O.; Kremer, K.; Grest, G. S. J. Chem. Phys. 1988, 88, 3286). For the first system, $\lambda=5$, the stable crystalline under those conditions is bcc and fcc is metastable. Fcc becomes stable for $\tilde{T}<2.365 \times 10^{-3}$ (Hoy, R. S.; Robbins, M. O. Phys. Rev. E 2004, 69, 056103). On the other hand, for the second system, $\lambda=10$, we have the inverse situation: the stable crystalline structure is fcc and bcc is metastable. These two systems allow us to compare the molecular mechanisms underlying crystal nucleation and growth when the bcc crystal is the stable phase or when the fcc crystal is the stable phase.

\section{Identification of the structural identity of the particles}

We describe here how we analyze the structure of the crystal nucleus and how we identify the structural identity (fcc, hcp or bcc) of each particle. We first briefly outline how we distinguish between solid-like and liquid-like particles (this is done according to the method proposed in ref. 10: ten Wolde et al., J. Chem. Phys., 104, 9932 (1996)). For a given molecule $i$, local orientational order parameters $\bar{q}_{l m}(i)(m=-l \ldots l)$ are defined according to

$$
\bar{q}_{l m}(i)=\frac{\sum_{j} Y_{l m}\left(\hat{\mathbf{r}}_{i j}\right) \alpha\left(r_{i j}\right)}{\sum_{j} \alpha\left(r_{i j}\right)}
$$

where $Y_{l m}\left(\hat{\mathbf{r}}_{i j}\right)$ is a spherical harmonics, $\alpha\left(r_{i j}\right)$ a weight function $\left(\alpha\left(r_{i j}\right)=\left(r_{i j}-r_{q}\right)^{2}\right.$ for $r_{i j}<r_{q}$ and $\alpha\left(r_{i j}\right)=0$ elsewhere). $j$ denotes a molecule which belongs to the first coordination shell of molecule $i$ and $\mathbf{r}_{i j}$ the vector joining the centers of mass of molecules $i$ and $j\left(r_{i j}\right.$ stands for the norm of this vector and $\hat{\mathbf{r}}_{i j}$ the corresponding unit vector). $r_{q}=1.4 \sigma$, corresponds to the first minimum of $\mathrm{g}(\mathrm{r})$ in the fcc crystal of the Yukawa crystal. 
We now define the normalized 13-dimensional $(m=-6, \ldots, 6)$ complex vector $\mathbf{q}_{6}(i)$ of components $\tilde{q}_{6 m}(i)$ defined as

$$
\tilde{q}_{6 m}(i)=\frac{\bar{q}_{6 m}(i)}{\left(\sum_{m=-6}^{6}\left|\bar{q}_{6 m}(i)\right|^{2}\right)^{1 / 2}}
$$

The dot product of the vectors $\mathbf{q}_{6}$ of two neighboring molecules $i$ and $j$ is

$$
\mathbf{q}_{6}(i) \cdot \mathbf{q}_{6}(j)=\sum_{m=-6}^{6} \tilde{q}_{6 m}(i) \tilde{q}_{6 m}^{*}(j)
$$

Two molecules are considered to be connected if $\mathbf{q}_{6}(i) \cdot \mathbf{q}_{6}(j)$ is larger than 0.5 i.e. if their local order parameters are correlated. We find that the number of connections per molecule rarely exceeds 8 in the liquid phase (less than $1 \%$ are identified as solid-like) and is seldom below 8 in both solid phases. A molecule connected to at least 8 molecules of its first neighbors is considered as solid-like and liquid-like otherwise.

We now explain how we distinguish between fcc-like, bcc-like and hcp-like molecules. The orders parameters considered in this analysis (i.e. $q_{4}$ and $\hat{w}$ ) are defined from the local order parameters $\bar{q}_{l m}(i)$ as

$$
\begin{gathered}
q_{l}(i)=\left[\frac{4 \pi}{2 l+1} \sum_{m=-l}^{l}\left|\bar{q}_{l m}(i)\right|^{2}\right]^{1 / 2} \\
\hat{w}_{l}(i)=\frac{w_{l}(i)}{\left[\sum_{m=-l}^{l}\left|\bar{q}_{l m}(i)\right|^{2}\right]^{3 / 2}}
\end{gathered}
$$

where

$$
w_{l}(i)=\sum_{\substack{m_{1}, m_{2}, m_{3} \\
m_{1}+m_{2}+m_{3}=0}}\left(\begin{array}{ccc}
l & l & l \\
m_{1} & m_{2} & m_{3}
\end{array}\right) \times \bar{q}_{l m_{1}}(i) \bar{q}_{l m_{2}}(i) \bar{q}_{l m_{3}}(i)
$$

We compute the distributions for the two local order parameters $q_{4}$ and $\hat{w}_{4}$ for the three crystalline structures fcc, bcc and hcp. On the basis of theses distributions, we identify a particle $i$ as

- fcc-like if $q_{4}(i)>0.16$ and $\hat{w}_{4}(i)<-0.05$,

- bcc-like if $q_{4}(i)<0.08$ or if $q_{4}(i)<0.11$ and $\hat{w}_{4}(i)<-0.025$,

- hcp-like if $q_{4}(i)>0.1$ and $\hat{w}_{4}(i)>0.04$.

These rules are summarized in figure 1 . We use the same rules for $\lambda=5$ and $\lambda=10$ 


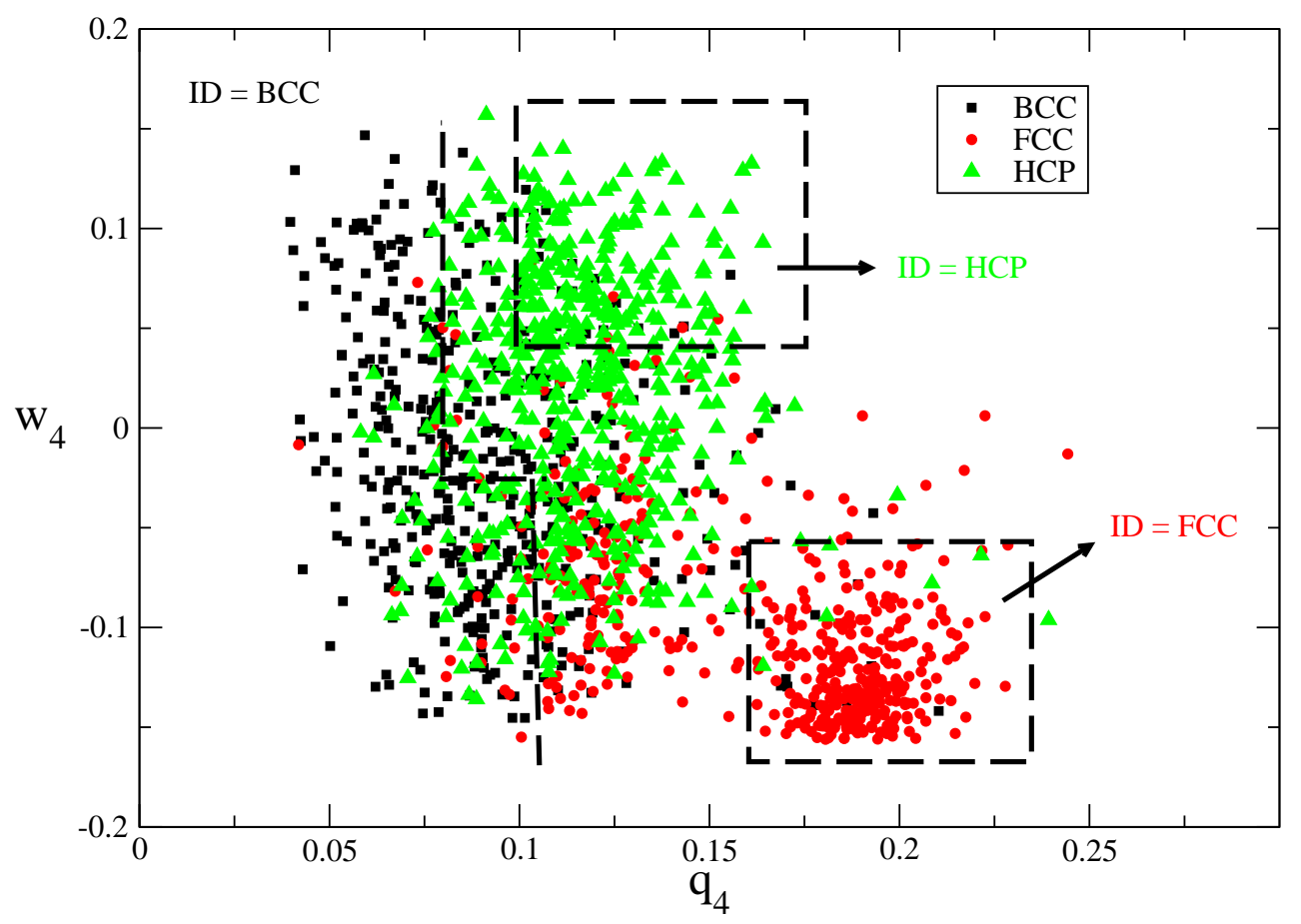

FIG. 1: Rules used to determine whether a particle is fcc-like, bcc-like or hcp-like $(\lambda=5)$.

As shown on Fig.1, we did not try to assign a specific structure to a molecule where there was a significant overlap between the distributions of local order parameters for two different structures. We therefore made a conservative choice. This is because we prefer being positive on the structural identity of a given particle and leave the identity of some particles as undetermined. We find this analysis satisfactory since it allows us to identify $80 \%$ of the structure inside the crystal nucleus. 